\title{
De musas, impostoras y tejedoras: la propiedad intelectual desde una perspectiva feminista
}

\author{
Lucero Ibarra Rojas*
}

\begin{abstract}
RESUMEN
Las críticas feministas al derecho han insistido en que el dominio masculino del contenido y la aplicación del derecho le han imprimido un sesgo machista de carácter histórico y, en cierta medida, inherente a las formas jurídicas. Sin embargo, se han abordado más bien poco los derechos de propiedad intelectual. ¿Qué indagación requerimos hacerle a la propiedad intelectual desde una perspectiva feminista? El objetivo de este texto es precisamente ofrecer una perspectiva feminista de la propiedad intelectual que explique tanto los elementos con los que esta da forma a los campos del arte y de la ciencia, como los sesgos de género que subyacen y a su vez se reproducen en estos campos y en las formas de la propiedad intelectual que les corresponden.
\end{abstract}

Crítica feminista; derechos de autor; patentes

\author{
Muses, impostores and embroiderers: \\ intellectual property from a feminist perspective
}

\begin{abstract}
Feminist critique of law has insisted that the masculine domination over law has imprinted it with a male chauvinist historical essence in its content and application, as well as, to some extent, in the legal form. However, intellectual property rights have rarely been referred to in this critique. What kinds of questions do we need to make to intellectual property from a feminist perspective? The aim of this article is precisely to offer a feminist perspective of intellectual property that explains both the elements through which it shapes the fields of art and science, as well as the gender bias that underline and are reproduced in these fields and in the corresponding intellectual property tools.
\end{abstract}

Feminist critique; copyright; patents

* Licenciada en Derecho, Universidad Michoacana de San Nicolás de Hidalgo, México. Maestra en Sociología Jurídica, Instituto Internacional de Sociología Jurídica de Oñati, España. Doctorada en Derecho y Sociedad por el programa Renato Treves de la Universidad de Milán, Italia. Profesora-Investigadora Titular de la División de Estudios Jurídicos del Centro de Investigación y Docencia Económicas (CIDE), México. ORCID: 0000-0002-5245-3189. Correo electrónico: lucero.ibarra@cide.edu

Artículo recibido el 8.2.2021 y aceptado para su publicación el 9.6.2021. 


\section{INTRODUCCIÓN}

$\mathrm{D}$ entro de la categoría de derecho privado se engloban aspectos jurídicos que son esenciales para la configuración jurídico-social del mundo, pero en las facultades de derecho, por lo menos en América Latina, estas materias suelen ser de las más tradicionales y apegadas al modelo positivista. Efectivamente, mientras que las visiones críticas del derecho suelen encontrar su espacio en materias más evidentemente interdisciplinarias, como las de sociología o filosofía del derecho, las materias de derecho privado suelen mantener una visión más purista acotada a la repetición dogmática de la ley, identificada como el "verdadero" derecho ${ }^{1}$. Esto no significa que no exista una fructífera academia feminista en diversos aspectos del derecho privado. Al contrario, en esta área del derecho se encuentran discusiones torales para la reflexión feminista, como pueden ser el acceso a la propiedad o las leyes que determinan las estructuras familiares; acerca de estas se ha reflexionado tan ampliamente y de tantos temas particulares que cualquier referencia en este punto sería insuficiente. No obstante, un comentario común en los intentos críticos de rediseño de mallas curriculares ${ }^{2}$ es que la intervención de estas materias es uno de los retos más grandes, el que frecuentemente queda solo como una buena intención. Dichos esfuerzos con frecuencia encuentran límites en visiones muy apegadas a una visión técnica de los cursos que tiende a verles como una herencia esencializada del derecho romano. Lo anterior significa un problema toral para una agenda progresista del derecho porque en el derecho privado se encuentran diversos elementos constitutivos del derecho, como la forma en la que el derecho produce conceptos, categorías o ideas que dan existencia y forma a las organizaciones y las instituciones sociales ${ }^{3}$.

En el derecho privado se configuran también lo que llamamos arte y ciencia, y las personas a las que se puede llamar autor o creador, mediante la propiedad intelectual. La propiedad intelectual refiere a una diversidad de formas jurídicas por medio de las cuales las ideas y los símbolos son asumidos en lo jurídico por modelos de apropiación que son bastante sui generis. El modelo de propiedad que se establece constituye diversas formas de monopolio que permite a quien ostenta el derecho (o a los Estados, en el caso de algunas formas de denominaciones de origen) determinar las condiciones de acceso y reproducción de los productos del intelecto humano, generando una escasez

${ }^{1}$ Véase: Ibarra, 2012. Lista, 2012.

2 Diversas actividades me han llevado a conocer iniciativas de este tipo al menos en la Universidad Intercultural Indígena de Michoacán (UIIM), la Universidad Autónoma de la Ciudad de México (UACM) y el Centro de Investigación y Docencia Económicas (CIDE), en México, y en la Universidad de Antioquia en Colombia.

${ }^{3}$ Edelman y Stryker, 2005, identifican tres 'ambientes jurídicos' como tipos ideales para identificar cómo el derecho determina las acciones de los actores económicos: el ambiente facilitador que incluye procedimientos a instancias para la gestión de las actividades y la resolución de conflictos; el ambiente regulatorio, que se compone de "reglas sustantivas que imponen autoridad social sobre varios aspectos de la vida organizacional” EdELmAN y STRIKer ,2005, p. 537; y el ambiente constitutivo, que "provee posibilidades cognitivas y valores que influencian la estructura, la forma y las estrategias de las organizaciones” EDELMAN y STRIKER, 2005, p. 540. [Traducciones de la autora]. 
artificial de intangibles que, por su naturaleza, son susceptibles de una amplia circulación sin agotarse ${ }^{4}$. Una canción, una novela, una invención, pueden ser reproducidas y, de hecho, esto potencia su valor; pero la función de los derechos de propiedad intelectual es establecer reglas que impongan costos monetarios a quienes desean acceder a estos productos.

La propiedad intelectual, ampliamente reconocida por su importancia para la economía, determina también las formas en las que el arte y la ciencia pueden ser bienes privados a pesar de que estén presentes en nuestro entorno. En la introducción a su libro The cultural life of intelectual properties Rosemary Coombe ${ }^{5}$ relata una caminata en Toronto resaltando los múltiples encuentros y desencuentros con formas de propiedad intelectual. Algo similar hace Siva Vaidhyanathan ${ }^{6}$ cuando describe una sucursal de una importante cadena de café estadounidense. La propiedad intelectual se encuentra presente aunque pasa inadvertida. Lo que también pasa inadvertido es que si la propiedad intelectual da nombre y forma a los procesos creativos, esta es, a su vez, determinada por procesos sociales y concepciones de género y de raza que también pasan inadvertidas. A pesar de lo anterior, y de algunos esfuerzos por construir perspectivas feministas en el área ${ }^{7}$, especialmente en el norte global, el sistema de derecho de propiedad intelectual ha sido poco referido en la crítica feminista al derecho.

Apenas en 2018, la Organización Mundial de la Propiedad Intelectual (OMPI) dedicó el Día Mundial de la Propiedad Intelectual al tema "Artífices del cambio: las mujeres en la innovación y la creatividad”, destacando la importancia de las mujeres en el ámbito de la propiedad intelectual, pero reconociendo también la necesidad de promover una mayor participación. Efectivamente, las mujeres hemos transitado la mayor parte de la historia de la humanidad sin tener posibilidades de instrucción o de tenerlas en menor medida que los varones de las mismas condiciones de raza y clase. La entrada de las mujeres a la mayor parte de las profesiones está marcada como un hito histórico y, de hecho, también por voces de varones que argumentaban la imposibilidad intelectual/biológica/social para que las mujeres se incorporaran al trabajo; podemos encontrar ejemplos de esto en la ciencia ${ }^{8}$, en el arte ${ }^{9}$ y en el ejercicio del derecho ${ }^{10}$.

Debido a que las condiciones históricas que han determinado el acceso a los distintos espacios de la vida pública nos proveen de buenas explicaciones para entender las brechas de género en el campo del arte y la ciencia que se documentan en cada una de las siguientes secciones, ¿qué indagación requerimos hacerle desde una perspectiva feminista a la propiedad intelectual?

\footnotetext{
${ }^{4}$ Véase: Drahos, 1996. Haunss, 2015.

5 COOMBe, 1998, p. 1-5.

${ }^{6}$ VAIDHYANATHAN, 2017, p. 1-6.

7 Véase: Barwa y Rai, 2003. Burk, 2007. Halbert, 2006. Swanson, 2015.

${ }^{8}$ Véase: BARWA y Rai, 2003. CODE, 1991.

9 Véase: Halbert, 2006. Paliyenko, 2016.

${ }^{10}$ Por ejemplo: Boigeol 2005.
} 
Las críticas feministas al derecho han insistido en que el dominio masculino del contenido y la aplicación del derecho le han imprimido un sesgo machista de carácter histórico y, en cierta medida, inherente a las formas jurídicas ${ }^{11}$. Esto significa que, más allá de los mandatos que claramente han representado herramientas de opresión de género instauradas en el derecho, es necesario analizar las formas jurídicas con una perspectiva de género que permita dilucidar sesgos o inercias de carácter patriarcal que son menos evidentes. Efectivamente, la postura liberal que promueve la participación de la mujer en espacios que han sido dominados por varones sin cuestionar los supuestos que subyacen a la construcción del derecho, o del conocimiento, pierden de vista elementos clave que perpetúan ese dominio. A las formas del derecho les subyacen supuestos sociales que corresponden a espacios y tiempos específicos, también a dinámicas de poder específicas, que acaban escondidas bajo el manto de postulados normativos que se afirman neutrales, universales e indiscutibles. Esto es una parte esencial del derecho y debería de serlo del análisis jurídico, y es también lo que posibilita la violencia simbólica ${ }^{12}$ del derecho, entendida como la pretensión de universalidad y objetividad que los juristas adjudican al derecho para fundamentar una obediencia sin cuestionamientos.

El objetivo de este texto es precisamente ofrecer una perspectiva feminista de la propiedad intelectual que explique tanto los elementos con los que esta da forma a los campos del arte y de la ciencia, como los sesgos de género que subyacen y a su vez se reproducen en estos campos y en las formas de la propiedad intelectual que les corresponden. En este sentido, se plantea una discusión sociojurídica en la que se analiza el fenómeno jurídico en sus dinámicas sociales con referencia a las construcciones en la letra de la ley.

Para lo anterior, tomaré como referencia la clasificación tradicional de la propiedad intelectual, abordando, en primer lugar, lo correspondiente al derecho de autor y, en segundo lugar, analizando las patentes como formas representativas de la propiedad industrial. Posteriormente, abordo el campo del conocimiento tradicional como un espacio de exclusión de las visiones más dominantes de la propiedad intelectual y donde una mirada interseccional nos muestra cómo ciertos rasgos de raza y clase configuran esta exclusión. Aunque usaré principalmente referencias del derecho mexicano, que es donde ubico mi trabajo, es importante notar que las observaciones son comunes a las herramientas de la propiedad intelectual en la mayor parte de las jurisdicciones nacionales y en las disposiciones internacionales. Finalmente, este trabajo concluye destacando la manera en la que la propiedad intelectual reproduce estructuras de género de la sociedad y, como alternativa, busco delinear algunas de las apuestas de la agenda feminista en la propiedad intelectual, particularmente en relación con el trabajo colaborativo y los modelos de acceso abierto.

\footnotetext{
11 Véase: Pateman, 1995. Ricoy, 2015. Swanson, 2015.

12 Bourdieu, 2003, p. 4.
} 


\section{EL AUTOR Y LAS MUSAS}

“¿Es necesario que las mujeres estén desnudas para entrar la Museo Met?” 13 . Como denunciaba en 1989 el colectivo Guerrilla Girls, menos del 5\% de las artistas con obras en la sección de arte moderno del Museo Metropolitano de Arte (Met) en Nueva York eran mujeres, mientras que las mujeres representaban el $85 \%$ de los desnudos en el mismo espacio. Lamentablemente, en un estudio reciente ${ }^{14}$ que analiza las obras de las colecciones permanentes de los principales museos de arte en Estados Unidos, con alrededor de diez mil artistas, se muestra, mediante el análisis de sus características demográficas, que la brecha de género sigue vigente: 75,7\% hombres blancos, 10,8\% mujeres blancas, 7,5\% hombres asiáticos y 2,6\% hombres hispanos; mientras que el resto de los grupos representados no alcanzaron ni siquiera el $1 \%$. Parece poco aventurado decir que esto no es exclusivo de los museos de arte estadounidenses. En lo que refiere concretamente al derecho de autor, aunque no fue posible encontrar las estadísticas de registros para México, los estudios de otros países son desalentadores. Un estudio pertinente a los derechos de autor en Chile muestra que los registros realizados únicamente por hombres constituyen el $55 \%$, mientras que aquellos realizados únicamente por mujeres son apenas el $15 \%{ }^{15}$. En Estados Unidos, los autores representan $66,63 \%{ }^{16}$ de los registros de copyright.

Esta realidad no se produce separada del derecho, sino que se relaciona con las dinámicas sociales en las que se concibe el derecho de autor. Efectivamente, el derecho de autor ${ }^{17}$ corresponde a un ideal producido en un momento histórico con condiciones económicas, sociales y de poder específicas. La noción del "autor", en un sentido moderno, se identifica como el producto de ciertas transformaciones económicas de los siglos XVIII y XIX ${ }^{18}$. Particularmente, respecto de la emergencia de individuos que hicieron de la labor literaria el sustento de sus vidas y la transferencia de la atención de los textos a estos individuos; es decir, la figura del autor como profesión. Esto evidencia la medida en la que las condiciones para el ejercicio de la labor creativa se han encontrado ligadas a ciertas condiciones de clase y de género. Aunque sí existen creadores reconocidos antes de estas transformaciones, en estos siglos se dio la confluencia de dos circunstancias que no se habían presentado de la misma manera anteriormente: que las necesidades económicas de los autores no se encontraran plenamente cubiertas por las condiciones

13 Guerrilla Girls, 1989.

14 Topaz et al., 2019.

15 Araya et al., 2012, p. 7.

16 Brauneis y Oliar, 2018, p. 129.

${ }^{17}$ En tradiciones anglosajonas, el campo de lo artístico se encuentra regulado en el modelo del copyright. Aunque alguna de la literatura que retomo en mi análisis se refiere a la tradición de copyright y, efectivamente, se pueden establecer varios paralelismos entre este modelo y el de derechos de autor, es importante tener presente que existen diferencias fundamentales, especialmente en lo que respecta al reconocimiento de derechos morales (explicados más adelante en este texto), que no son contemplados explícitamente por el modelo de copyright.

${ }^{18}$ Véase: Craig et al., 2011. Halbert, 2006. Woodmansee, 1984. 
de su clase social y que estos autores tuvieran las condiciones sociales para influenciar los marcos jurídicos y llevar sus intereses a la letra de la ley.

El ideal que se posicionó de manera consolidada ya en el siglo XIX era el del creador como individuo excepcional quien, de manera solitaria e innovadora, producía una obra única, posible solamente gracias al mérito y las características individuales de su creador $^{19}$. En épocas previas, los productos circulaban de una manera más libre y prevalecían las formas más colaborativas de escritura ${ }^{20}$. A partir de las incursiones del lobby de diversos autores románticos, el arte concibe como un producto de una introspección del autor, quien fundamenta la legitimidad de su derecho en la excepcionalidad de su obra que está relacionada directamente con la excepcionalidad de su propia persona ${ }^{21}$. En este proceso la historia de las ideas dio un giro marcadamente individualista ${ }^{22}$. Al reforzarse el vínculo jurídico y social de la obra en relación con el autor, se consolida también la forma jurídica como propiedad privada y el énfasis en la originalidad como eje para establecer el valor de la obra. El criterio de originalidad, a su vez, se transfiere de la obra al autor mismo; es decir, la obra se ostenta como original no en virtud de sí misma, sino en relación con el autor.

El derecho de autor, en la actualidad, constituye una forma de monopolio que se encuentra amparado por el Estado y permite a quienes ostentan los derechos (que no siempre son los autores, o no lo son de todos los derechos) recibir un incentivo económico por la explotación de su obra, así como controlar algunos aspectos de esta explotación. Atendiendo a la construcción del autor antes descrita, este monopolio se configura por dos elementos complementarios. Por un lado, se tienen los derechos morales, que refieren de manera directa al vínculo inexorable entre autor y obra, que debe ser reconocido de manera permanente. Cuando una persona compra un cuadro, el objeto le pertenece, pero no así el derecho de ostentarse como autor. Los derechos morales no son susceptibles de ser vendidos, ni de transmitirse de ninguna otra manera, e incluyen otros aspectos relacionados con el prestigio y la integridad de los autores. Por el otro lado, están los derechos patrimoniales que son los que adjudican beneficios económicos a las personas que los ostentan, además de la posibilidad de controlar y beneficiarse de la exhibición de la obra. A diferencia de los derechos morales, los derechos económicos se pueden transmitir de diversas formas y tienen una temporalidad limitada, aunque esta se ha ampliado considerablemente durante los últimos cien años en buena parte del mundo ${ }^{23}$. Esta configuración del derecho de autor posibilita la estructura comercial del

${ }^{19}$ Véase: Burk, 2007. Craig et al., 2011; Halbert, 2006. Jaszi y Woodmansee, 1994. Woodamnsee, 1984.

20 Véase: WoOdAMNSEE, 1994.

${ }^{21}$ Drahos y Braithwaite, 2002, p. 33.

22 Véase: Foucault, 1998.

${ }^{23}$ México, reformó su legislación en la materia como resultado de las negociaciones del Tratado de Libre Comercio de América del Norte (TLCAN) a finales del siglo pasado (Aboites y Soria 2008). Actualmente, los derechos patrimoniales tienen una vigencia de cien años después de la muerte del autor; siendo uno de los países donde el derecho se encuentra vigente por más tiempo. 
sector creativo, porque permite el dominio que las industrias intermediaras, como las editoriales o las disqueras, han adquirido respecto del manejo de derechos patrimoniales en lo que se conoce como derechos conexos.

Sin embargo, en su forma actual, la estructura de los derechos de autor también construye un nicho moral para el ideal decimonónico del autor. El derecho está centrado precisamente en el autor, se sigue anclando en su mérito y más que encontrar criterios para que la obra de alguna manera "merezca" la "protección”. Se construye discursivamente; es una estructura de recompensa para quien ejerce la labor creativa y a su originalidad. Las leyes relativas al derecho de autor, en realidad, hablan poco del arte o de los valores estéticos que hacen que un objeto creativo amerite el nombre; pero hablan del creador, de cómo identificar sus contribuciones, de sus méritos morales. En el caso de México, por ejemplo, el artículo 5 de la Ley Federal del Derecho de Autor establece explícitamente que la protección es independiente del mérito de la obra y el artículo 13 señala que el derecho es un "reconocimiento que hace el Estado en favor de todo creador". Asimismo, uno de los criterios que establece la ley para clasificar las obras es en función del autor (conocido, anónimas y pseudónimas, según el artículo $4^{\circ}$ ) y la clasificación de obras entre colectivas o de colaboración depende de que se pueda identificar la contribución de cada autor. Incluso, hasta hace muy poco, esta ley contemplaba que si no se podía identificar el autor individual de una obra, la misma quedaba en el dominio público, es decir, se podía explotar libremente. Estas obras se identificaban, además, con las creaciones de los pueblos y comunidades indígenas en México.

La posición del autor es también una posición de autoridad ${ }^{24}$. Históricamente esto ha significado que la versión del mundo y, por tanto, la construcción del mundo, en gran medida ha dejado de lado a las mujeres. El hecho de que la voz y la mirada pertenezca de manera dominante a un sector social, como el de los hombres blancos, representa también la articulación de una dinámica de $\operatorname{poder}^{25}$. La protección del derecho de los autores, debido a la distribución de las dinámicas de poder asociadas con los productos culturales reconocidos como tales, ha sido una manera de perpetuar jerarquías que se encuentran ocultas $y$, sin embargo, son sumamente significativas ${ }^{26}$.

Lo anterior es aún más evidente si tomamos en cuenta el otro rol tradicional de las mujeres en los procesos creativos: como apoyo invisible o inspiración. La construcción del autor individual y el mérito de su originalidad esconde los procesos sociales en los que la vida y la creación toman lugar, y también el papel de las mujeres que han sido parte de estos procesos sin gozar del privilegio del uso de la palabra. Como indica Burk ${ }^{27}$, no es que las mujeres tuvieran poco que decir, sino que su voz no tenía espacio y, en el espacio íntimo en el que se desarrollaban al ser excluidas de lo público, las ideas eran apropiadas por varones que gozaban del espacio y la autoridad de sus voces, a pesar de

\footnotetext{
${ }^{24}$ Foucault, 1998, p. 307.

25 Véase: BARWA y RAI, 2003.

26 SwANSON, 2015, p. 183-185.

${ }^{27}$ Véase: Burk, 2007.
} 
cualquier proceso de socialización en el que tomara forma. Esta condición es precisamente la que evidenciaba el colectivo Guerrilla Girls cuando señalaba la estadística de artistas mujeres en comparación con los desnudos de mujeres en el $\mathrm{Met}^{28}$, pero se presenta en diversas expresiones artísticas. En la literatura, este fenómeno es evidente en casos como el de Zelda Fitzgerald ${ }^{29}$, aunque muchos otros y diversas formas de apoyo se pueden señalar como ejemplos ${ }^{30}$. Algunas de las formas en las que se identifica este apoyo son actividades, como a edición y corrección de textos, que previamente se consideraban parte constitutivas de la obra literaria ${ }^{31}$.

En este proceso, las condiciones históricas de participación de las mujeres en el campo del arte han generado una objetivación de lo femenino y su reificación en los roles más tradicionales de género. Efectivamente, la concepción misma de lo femenino ha sido frecuentemente dicha por hombres. Sontag ${ }^{32}$, en su crítica al arte, discute la manera en la que la noción de belleza se ha construido determinada también por dinámicas de género. La belleza es asignada como valor supremo a las mujeres, que después son juzgadas por sus esfuerzos por mantenerla. La belleza es también una categoría conectada con la naturaleza, con lo banal y lo caprichoso. La belleza es entonces un mérito que se concibe como valioso en la medida que debe poco al esfuerzo de la persona, es un mérito natural sin mérito personal. La objetivación de la mujer en el mundo del arte es frecuentemente una reificación de lo femenino en sus roles más tradicionales.

El enfoque en la originalidad del autor individual también tiene un efecto en los tipos de productos que son considerados en el derecho de autor, y los productos que caen fuera de su marco. Como en otros aspectos productivos, las mujeres somos reconocidas en la medida que generamos valor en espacios ocupados históricamente por hombres; mientras que los espacios más reconocidos como dominio de las mujeres se mantienen invisibles ${ }^{33}$. Las artes textiles y el campo de la moda, en general, frecuentemente no gozan de protección de derechos de autor ${ }^{34}$. Las expresiones artísticas que tienen un sentido utilitario, son descalificadas por sus elementos funcionales, que se conciben en oposición a la originalidad y el valor puramente estético, pero también son las expresiones donde se ha dado más el trabajo de mujeres. Así, el modelo de propiedad intelectual que se basa en el genio del autor y desconoce las creatividades que son colectivas o conectadas con la tradición, desfavorece entornos creativos colectivos, que también son frecuentemente dominados por mujeres.

En este punto, es importante destacar que no se trata de que ciertos espacios o disciplinas sean inherentemente masculinos y otros femeninos, sino que estas divisiones dependen de las configuraciones históricas de nuestras sociedades. La labor de destacar

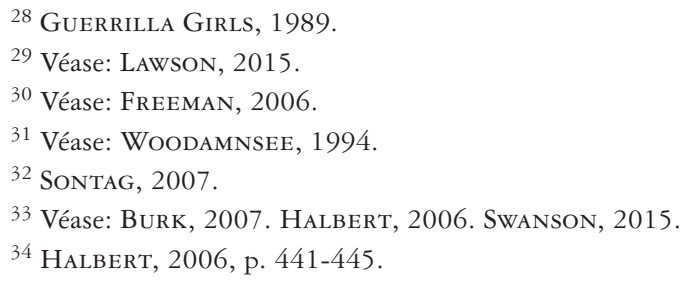


los espacios que han sido ocupados por mujeres, frecuentemente se encuentra con la tentación de presentar una visión fundamentalista de los roles de género y, aun con una intención de restaurar el valor a esos espacios, se puede reencasillar lo femenino en varias características que se construyen en oposición a lo masculino ${ }^{35}$. Entonces, más que producir una mirada que idealice los roles tradicionales, la intención es mostrar que estos roles efectivamente se han caracterizado por formas de trabajo y creatividad que no han encontrado lugar en el derecho.

En contraparte, mostrar los espacios que han quedado fuera del derecho nos puede mostrar deficiencias importantes de la forma jurídica del autor. El trabajo creativo no ocurre verdaderamente aislado de sistemas sociales y dinámicas de convivencia; incluso los genios tienen familias, amistades y colegas ${ }^{36}$. Las industrias creativas contemporáneas presentan una amplísima diversidad de contribuciones de diversos tipos que hacen posibles los productos y que, sin embargo, son consideradas insignificantes en la tradición romántica del autor; incluyendo una amplísima variedad de trabajos que suelen presentarse como una modalidad de la palabra editor.

\section{LA CIENCIA ES LO QUE HACEN LOS CIENTÍFICOS}

La construcción del autor en el ideal decimonónico que ha sido determinante para dar forma al derecho de autor es replicada también en la forma más representativa de la propiedad industrial, que es la patente ${ }^{37}$. Una patente es una forma de registro de conocimiento con el que se adquiere el monopolio de la explotación de este por un tiempo determinado. Las patentes representan una limitante a la competencia económica y se encuentran justificadas en el mérito del desarrollo científico y tecnológico, así como en la necesidad de un incentivo económico que motive a las personas. No resulta particularmente evidente en su planteamiento la conexión con la forma individualista del autor y, de hecho, los requisitos esenciales de las patentes sí parecen centrarse en el producto más que en la persona: las patentes se otorgan a quienes registren innovaciones que, como tales, representen un distanciamiento del estado de la ciencia y que tengan un uso práctico.

Sin embargo, la forma jurídica de las patentes retoma los ideales de la ciencia positiva y, por tanto, planteamientos epistémicos que corresponden a una posición histórica y social, también determinada por los roles de género. Efectivamente, la construcción cartesiana del conocimiento se basa en la existencia de un individuo guiado por la razón, solitario

${ }^{35}$ CODE, 1991, p. 17.

36 Véase: Freeman, 2006.

${ }^{37}$ Bajo la denominación de propiedad industrial encontramos una variedad de herramientas. Algunas de estas están relacionadas con la apropiación de conocimiento útil para el desarrollo industrial, pero también encontramos otras figuras, como las marcas, en donde lo que se apropia son símbolos o imágenes que tienen valor para el comercio. En este trabajo me enfoco solamente en las primeras. 
y autosuficiente en su búsqueda de conocimiento ${ }^{38}$. Por fuera del sujeto que conoce, se encuentra el objeto a ser conocido, y el proceso de conocerlo es uno de apropiación por parte del científico. En este proceso, el valor de la objetividad es clave, por lo que el contexto de quien conoce debe ser irrelevante y el proceso de conocer supone no tener impacto alguno. La separación del sujeto que investiga de quienes son parte protagónica del fenómeno estudiado no se trata solamente de un proceso de objetivación en pro de un ideal de objetividad, es también un proceso que reafirma una dinámica epistémica de poder. Esta dinámica, como explicaba Foucault, es la expresión del poder que se confiere al autor en el campo de lo científico y, de la misma manera, ubica al científico en el lugar del conocedor y al resto del universo en el lugar pasivo de ser susceptible de ser conocido; de ser, en este proceso, definido. Esta forma de conocer, al conformarse como paradigma dominante de la ciencia y el derecho, se vuelve una epistemología caníbal ${ }^{39}$, que niega la validez de otras formas de conocer.

Asimismo, los rasgos asociados con la ciencia han sido identificados históricamente como inherentes al hombre. Efectivamente, más de un filósofo se ha encargado de adjudicar al hombre el dominio de la razón, mientras las mujeres hemos sido relegadas a ser dominadas por las emociones ${ }^{40}$. Y a causa de que la ciencia positivista ha construido el intelecto y la emoción como opuestos, en buena parte de la historia de la humanidad las mujeres no hemos sido concebidas como personas capaces de generar conocimiento. La descalificación o la visión limitada de las posibilidades intelectuales de las mujeres incluso ha referido a supuestas características biológicas que luego han sido desmentidas.

$\mathrm{Al}$ igual que en el campo artístico, los espacios de conocimiento de las mujeres han sido oscurecidos. Conocimientos en ámbitos como el culinario, la maternidad o incluso ciertas formas de medicina que se practican en lo doméstico frecuentemente replican estándares de las ciencias que se sostienen objetivas: son probados en una variedad de condiciones, tienen aplicaciones prácticas evidentes y pruebas empíricas constantes ${ }^{41}$. Y, aun así, estos conocimientos se mantienen al margen del apelativo de ciencia y tampoco se pueden registrar como patentes.

Un ejemplo de esto es que en algunas legislaciones se pueden patentar olores y normalmente se pueden registrar como marcas, pero la cocina, el campo de los sabores, encuentra más obstáculos. De hecho, si prestamos atención a la definición de invención en la que se basan las patentes en la Ley Federal de Protección a la Propiedad Industrial de México, que hemos usado como ejemplo, el ámbito de la cocina sería fácilmente registrable como patente. En el artículo 46 de esta ley se señala que una invención es una creación humana, que transforme la materia, "para su aprovechamiento por el hombre y satisfacer sus necesidades concretas”. No obstante, no se encuentran registros de recetas de cocina como patentes en México.

\footnotetext{
38 Véase: Burk, 2007. CODE, 1991.

39 Teubner y Fischer-Lescano, 2008, p. 26-27.

${ }^{40}$ Code, 1991, p. 46-47.

${ }^{41}$ Véase: BARwa y Rai, 2003. Code, 1991.
} 
Lo anterior se encuentra también ligado a la falta de reconocimiento del trabajo que históricamente hemos realizado mujeres y que se encuentra circunscripto al espacio doméstico; alejado del espacio de producción que es no solamente público, sino también industrial ${ }^{42}$. Nuevamente, no se trata de afirmar que estas formas de conocer sean inevitable y fundamentalmente femeninas. Es decir, que se encuentran conectadas con un ser mujer estereotipado y, a veces, incluso biológicamente diferenciado. Al contrario, los espacios donde las mujeres han desarrollado un conocimiento que ha sido ignorado históricamente también se configuran socialmente.

Además, en el ámbito académico, la creatividad y el intelecto de las mujeres siguen enfrentando obstáculos enormes. Las mujeres ganamos menos, ocupamos puestos de menor perfil y pagamos costos frecuentemente irremediables en nuestras evaluaciones académicas por elegir la maternidad como parte de nuestras vidas ${ }^{43}$; de hecho, los mismos problemas se pueden encontrar en la profesión jurídica ${ }^{44}$. Aunado a esto, varios estudios han documentado las otras maneras sutiles ( $\mathrm{y}$ no tanto) en las que nuestro trabajo suele ser menospreciado: desde evaluaciones estudiantiles menos favorables ${ }^{45}$, menor presencia en citas ${ }^{46}$ y en las bibliografías básicas de los cursos, pasando por el peso excesivo en las labores administrativas ${ }^{47}$ y hasta el hecho de que la noción de síndrome de la impostora (como el miedo constante a ser descubierta como un fraude) fue acuñada en un estudio respecto de la psicología de mujeres en la academia ${ }^{48}$.

No es de sorprender que muchos de estos problemas se observen también en indicadores de ciencia y tecnología en el marco de la propiedad intelectual, como son las patentes. Diversos estudios documentan que existe una importante brecha de género en la producción de patentes ${ }^{49}$. En México, si bien la mayor parte del registro de patentes es efectuado por agentes extranjeros, principalmente industrias transnacionales, la brecha de género en las personas y equipos nacionales que registran patentes es abismal ${ }^{50}$. A pesar de todo lo anterior, el entorno académico, sin lugar a duda, goza de un amplio reconocimiento y legitimidad.

42 Véase: Barwa y Rai, 2003. HALBERT, 2006.

43 Véase, por ejemplo: White. 2015. Monroe et al. 2008.

${ }^{44}$ Véase, por ejemplo: MANZO ET. AL, 2016. POZAS Y RÍOS, 2021.

45 Véase: Mitchell y Martin, 2018.

46 Véase: MALINIAK et al., 2018.

47 Véase: Guarino y Borden, 2017.

48 Véase: Clance E IMEs, 1978.

49 Véase: Burk, 2015. Frietsch et al., 2009. Hoisl y Mariani, 2017. Hunt et al., 2013. Jensen et al., 2018. KAHLER, 2011.

50 Santamaria Hernández y Castro De Angel, 2016, p. 25-28. 


\section{LAS MUJERES COMO GUARDIANAS DE LA TRADICIÓN}

Si bien la mirada a los espacios más tradicionales de la propiedad intelectual, como los derechos de autor o las patentes, nos muestra la poca participación que históricamente han tenido las mujeres, en espacios delimitados por la instrucción, y los retos que se mantienen vigentes; también se enfoca en áreas que suelen ser privilegiadas y tener ciertas características, no solamente de género, sino también de raza y clase. Una de las más grandes aportaciones, más o menos recientes, de los estudios feministas y de género, ha sido la perspectiva interseccional que emerge de los feminismos racializados en Estados Unidos y es retomada por las agendas feministas decoloniales en América Latina $^{51}$. Desde esta perspectiva, se considera que una mirada que solamente toma en cuenta los procesos de violencia que están vinculados el género pierde de vista otros elementos que son determinantes en la experiencia de vida de las personas; por tanto, es necesario construir una mirada que tome en cuenta dimensiones de género, origen étnico y clase. El análisis interseccional no refiere simplemente a una suma de opresiones, como es frecuentemente caracterizado. La intención es identificar la forma que elementos de género, raza y clase interactúan en la experiencia de vida de las personas, pudiendo poner mayor o menor énfasis en cada elemento.

Esta perspectiva nos lleva a voltear la mirada al campo de las expresiones culturales tradicionales y los conocimientos tradicionales. Estos dos apelativos refieren a una larga acusación hecha a los modelos más tradicionales de propiedad intelectual por dejar de lado formas de conocimiento y creación que se encuentran ligadas a la tradición, son desarrolladas colectivamente y que no suelen establecer límites tan claros entre lo científico, lo creativo e incluso lo espiritual ${ }^{52}$. En la actualidad, la Organización Mundial de la Propiedad Intelectual (OMPI) ${ }^{53}$ identifica, por un lado, bajo el rubro de conocimientos tradicionales, los saberes, las habilidades y las prácticas de comunidades indígenas y otras comunidades tradicionales, curiosa y paradójicamente, también sus innovaciones, a pesar de que podrían entrar en el marco de la propiedad industrial. Por el otro lado, la $\mathrm{OMPI}^{54}$ señala que las expresiones culturales tradicionales se integran por las formas tangibles e intangibles en las que se expresan las culturas. Estas distinciones acercan un poco más el campo de lo tradicional a los modelos de propiedad intelectual mediante la distinción entre lo científico, como conocimiento, y lo artístico, como expresiones culturales. Sin embargo, los dos apelativos siguen refiriéndose a expresiones intelectuales que se mantienen fuera de los marcos de propiedad intelectual, o incluidos de maneras limitadas. Como se mencionó, en el caso de México, apenas en 2019 se cambió la ley de derecho de autor para que dejara de considerar a las expresiones de la cultura popular como parte del dominio público.

\footnotetext{
51 Véase: Crenshaw, 1989, 1991. Macleod, 2018. Masson, 2018.

52 Véase: Arup, 2009. Dommann, 2008. Gearhart-Sema, 2009.

53 WipO, 2017, p. 9-11.

${ }^{54}$ Idem.
} 
Aunque el campo del conocimiento y las expresiones culturales tradicionales se encuentra ampliamente estudiado, no se ha hecho un énfasis particular en las condiciones de género que lo caracterizan. En el caso de las artes indígenas, por ejemplo, las exploraciones a una variedad de tradiciones textiles y alfareras, principalmente, muestran campos dominados de manera significativa por mujeres, quienes también realizan labores domésticas y de crianza ${ }^{55}$. Sin embargo, apenas algunos trabajos acerca de propiedad intelectual y expresiones culturales tradicionales se posicionan desde una perspectiva feminista ${ }^{56}$. Muchas de las actividades creativas que encuentran un espacio en las expresiones y conocimientos tradicionales corresponden también a los espacios domésticos y alejados de los procesos industriales en los que las mujeres se han desarrollado con mayor frecuencia en distintas culturas ${ }^{57}$. Un claro ejemplo de esto, ya mencionado, es el área textil. El tejido, el bordado, lo relacionado en general con la vestimenta, han sido actividades eminentemente femeninas. Todas estas actividades se han concebido fuera de la propiedad intelectual y, cada vez más, como parte de las expresiones culturales tradicionales.

Un aspecto que es fundamental a considerar es que, a diferencia de muchos de los elementos que se encuentran englobados en el conocimiento y las expresiones culturales tradicionales, y que se caracterizan por ubicarse en entornos de origen indígena, el trabajo de las mujeres en el campo oscurecido de los textiles se encuentra de manera más transversal en distintas condiciones de origen étnico y de clase. La realización de edredones en la técnica que se conoce como quilt en la que se integran distintos tipos y técnicas de textiles, frecuentemente incluyendo bordados con historias o la refuncionalización de textiles, va posicionándose como una forma de expresión de historia de las mujeres ${ }^{58}$. Esta afirmación resuena con la consigna del feminismo decolonial que ve a los tejidos como "los libros que la Colonia no pudo quemar" 59 y que, en cierta forma, se deriva de las condiciones de género que son comunes en diversos aspectos del conocimiento y las expresiones culturales tradicionales.

Adicionalmente, este tipo de expresiones se encuentran dominadas por procesos colectivos de creación. En diversas tradiciones textiles el trabajo no solamente se realiza en grupos en los que cada persona realiza su trabajo en compañía de otras, sino que además diversas personas trabajan en la misma pieza. En otras ocasiones distintas personas se especializan en distintos pasos de la realización de una pieza, de manera que la obra final ha pasado por más de un par de manos. Las distintas formas de intercambio generan estéticas colectivas que se comparten y se transforman, conformándose como verdaderas tradiciones estéticas indígenas ${ }^{60}$. En todos estos casos, los procesos de

55 Véase: Bartra, 2005. Escobedo, 2019. Ibarra, 2016. Montes, 2020. Treviño y Coautoras, 2018. Zapata-Martelo y Suares-San Román, 2007.

\footnotetext{
56 Véase: Barwa y Rai, 2003. Gearhart-Sema, 2009.

57 Véase: Bartra, 2005. Halbert, 2006. Mithlo, 2019.

${ }^{58}$ Halbert, 2006, p. 443-445.

59 Véase: Toro, 2019.

${ }^{60}$ Véase: GARrido, 2015.
} 
aprendizaje y de realización de trabajos de valor estético también implican una variedad de conocimientos y otros aspectos de socialización vinculados con el género. Quizás por esto, la más reciente reforma de derecho de autor en México atribuye a las autoridades de las comunidades la facultad de autorizar los usos de las expresiones culturales de las comunidades indígenas. A pesar de la pertinencia de esta consideración, también es cierto que no se han contemplado las complejas dinámicas de género internas a las comunidades.

Esto se suma a contextos en los que, identificada una titularidad o mérito concreto, quede en manos de hombres. Si bien las piezas de arte indígena han entrado en dinámicas de identificación individual de artistas por medio de firma, apenas recientemente y en gran medida por la intervención de agentes externos a las comunidades ${ }^{61}$, hay otras dinámicas de reconocimiento que nos muestran esto. Por ejemplo, aunque las mujeres se encuentran ampliamente representadas en las expresiones culturales tradicionales, cuando observamos catálogos de arte popular, la identificación de personas excepcionales para ser nombrados maestros artesanos se vuelve atípicamente (para el campo de la artesanía) masculinizada. En el catálogo de Grandes Maestros del Arte Popular Mexicano ${ }^{62}$, los sectores con mayor participación de mujeres son el textil, con 70,5\% y las fibras, con $58,3 \%$, a pesar de que la participación de las mujeres en estos sectores es amplísima. También lo es en la alfarería, pero el catálogo solamente incluye 27,5\% de mujeres en este sector, mientras que en las otras disciplinas, la presencia de mujeres reconocidas como Maestra del Arte Popular es de $10 \%$ en el trabajo en madera, 5,9\% en metales y $8,6 \%$ en técnicas varias que incluyen papel, piel, arte plumario, pasta de caña, entre otras.

Una mirada interseccional nos hace ver que los valores de racionalidad han sido atribuidos históricamente al sector de la elite de los hombres blancos y que, a pesar de la generalidad del dominio patriarcal, sus prácticas son diferenciadas en función de raza y clase. Es bajo el apelativo de tradicional en donde se han puesto las formas de conocer y crear que se alejan del canon de la propiedad intelectual y donde las culturas de pueblos colonizados se encuentran ampliamente representadas ${ }^{63}$. Al mismo tiempo, una perspectiva interseccional a las sombras de la propiedad intelectual nos muestra que hay creatividades y conocimientos de mujeres que se encuentran aún más distantes de ser reconocidos, que la colonialidad de los modelos de propiedad intelectual afecta aún más a las mujeres y que una posible crítica feminista a estos modelos nos invita a repensarlos.

En la actualidad, la creación de marcos jurídicos relacionados con las expresiones culturales tradicionales es una preocupación que aqueja a muchos colectivos de mujeres indígenas en América Latina ${ }^{64}$. En general, la falta de marcos de protección para las expresiones culturales tradicionales y el conocimiento tradicional afecta de manera más significativa a los países del sur global, al tiempo que solidifica la hegemonía cultural

\footnotetext{
${ }^{61}$ IBARRA, 2016, p. 214-215.

62 Fernández de Calderón, 2001.

63 Véase: Dommann, 2008. Flórez, 2007.

${ }^{64}$ Véase: Treviño y CoAutoras, 2018.
} 
global de los productos del norte global garantizando los correspondientes intereses económicos ${ }^{65}$. Al mismo tiempo, el estudio de este campo creativo se encuentra caracterizado por procesos de despojo también efectuados por agentes del norte global frente a comunidades tradicionales, frecuentemente en labores que tradicionalmente son desarrolladas por mujeres ${ }^{66}$. Frente al despojo, se han realizado iniciativas que buscan dar forma a un nicho para las expresiones culturales tradicionales como parte de una reforma en materia de propiedad intelectual y también se han desarrollado iniciativas que buscan resaltar otras formas de crear y éticas de compartir, como se expresa en la declaración de la comunidad de Santa María Tlahuitoltepec (2015) frente a uno de los casos más notorios de réplica de diseños textiles tradicionales que se han dado en México ${ }^{67}$. Para estas iniciativas de reforma es central construir agendas que, lejos de aceptar irreflexivamente los paradigmas de autor y científico ampliamente definidos por un sector de raza y género específico, busquen retomar valores feministas de igualdad y dignidad ${ }^{68}$.

\section{A MANERA de CONCLUSión ¿UNA AgENDA FEMINISTA PARA LA PROPIEDAD INTELECTUAL?}

¿Qué aprendizajes podemos retomar entonces de esta indagación feminista a la propiedad intelectual desde una perspectiva sociojurídica? En el ejercicio que aquí se presenta de visibilizar las estructuras masculinizadas que subyacen a los modelos del derecho de autor y las patentes y el rol que hemos tenido las mujeres en los campos sociales que esta área del derecho regula, nos devela procesos de poder y las estructuras sociales que determinan la propiedad intelectual (Halbert 2006). Estas estructuras son parte de la forma de la ley, se relacionan con la centralidad del autor, con los productos registrables como patentes y con las expresiones que se han dejado fuera de la propiedad intelectual o con derechos limitados.

Así, a los problemas que enfrentamos las mujeres en los espacios que han sido considerados históricamente como masculinos, como la academia, se añade el completo oscurecimiento del valor de los espacios, las creatividades y los intelectos desarrollados en espacios que han sido considerados históricamente como femeninos. En este sentido, un ejercicio de verdadera valoración de las mujeres en la creatividad y la innovación requiere repensar los paradigmas de los modelos de propiedad intelectual y otros aspectos de regulación en donde el derecho configura lo que comprendemos como conocimiento, como arte y como ciencia. En este repensar, es fundamental dar un lugar a las críticas feministas y a las propuestas que se esbozan a partir de éticas de reconocimiento que no implican monopolios.

\footnotetext{
65 Véase: JASZi y WoOdMANSEe, 1994.

${ }^{66}$ Véase: Bartra, 2005. Barwa y Rai 2003; Gearhart-Sema, 2009. Oehlerich De Zurita, 1999.

67 Véase: IBARRA, 2019.

${ }^{68}$ Gearhart-Sema, 2009, p. 390-395.
} 
Sin embargo, es importante mantener presente la advertencia de que los espacios y las formas creativas que han sido dominadas históricamente por mujeres no lo son por una forma esencializada de lo femenino, sino por los procesos sociales y las formas de poder que las han configurado. Al pensar la propiedad intelectual, también debemos tener presente que el feminismo no es una sola corriente cuyos postulados y objetivos sean compartidos homogéneamente por todas las personas que se suscriben como feministas, lo que implica que no todas las posturas feministas necesariamente se constituirían como críticas del modelo de propiedad intelectual. La diversidad en experiencias creativas nos muestra efectivamente los límites de lo jurídico. En principio, no todas las ideas extraordinarias pertenecen al mercado, ni son los monopolios la única manera de reconocer el valor de una idea. Sin embargo, la falta de acceso a los derechos también se sostiene en una desvalorización racista y clasista del trabajo de muchas mujeres cuyas condiciones económicas son profundamente injustas. Aunque las muchas cooperativas de mujeres artesanas no son siempre, o no necesariamente, una expresión de armonía, sí son un reconocimiento de necesidades y creatividades compartidas. Asimismo, las herramientas de la propiedad intelectual no significan opresión para todas las mujeres y muchas creadoras y sus modelos creativos pueden encajar con los mandatos de los derechos de autor o las patentes; aunque, como se muestra en este trabajo, la brecha de género se mantiene presente. Sin embargo, la expectativa de intervenir los espacios y desarrollar alternativas es parte de más de una agenda feminista en los campos que conciernen a la propiedad intelectual.

A pesar de lo anterior, más que ofrecer una conclusión, me parece que la pregunta, que se mantiene abierta, es precisamente si la mirada feminista que se esboza en este trabajo puede ayudarnos a pensar otras alternativas. Y, en este sentido, considero que existen ya algunas aportaciones que merecen ser mencionadas. Las apuestas feministas de base colectiva se han caracterizado por acompañar visiones de derechos de autor que tienden a la apertura. Más que reivindicar los extensivos límites que impone a la circulación de productos culturales el derecho de autor, una perspectiva feminista nos invita a considerar las opciones de acceso abierto que se han desarrollado como parte de los esfuerzos de diversos colectivos para retomar el control de las formas de distribución de la cultura y potenciarla ${ }^{69}$. La teoría feminista desarrollada respecto de modelos de acceso abierto sostiene que las ideas no aparecen de manera independiente, ya que las personas crean en redes de relaciones sociales. Así, los derechos de autoras y autores tendrían que establecer reglas de interacción con los y las usuarias, en lugar de promover modelos de exclusión. En este mismo sentido, los entornos colaborativos han sido identificados como favorables para el desarrollo profesional de las mujeres en la ciencia y no es de sorprender que el impulso de metodologías colaborativas en investigación políticamente comprometida con pueblos indígenas, tenga también una marcada orientación feminista $^{70}$. Aunque la reflexión acerca de las implicaciones que esto podría tener para

\footnotetext{
69 Véase: Craig et al., 2011. Halbert, 2006. Gearhart-Sema, 2009.

${ }^{70}$ Véase: Macleod, 2018. Masson, 2018. Rappaport, 2018.
} 
derechos de autoría es más bien limitada, otras experiencias y planteamientos de cómo podría gestionarse la propiedad intelectual ${ }^{71}$, muestran muchas maneras de entender la creatividad y el conocimiento. También muestran que la identificación de las obras puede responder a distintos valores y estrategias de reconocimiento. En este sentido, la principal aportación de una mirada feminista es abrir una puerta; desnaturalizar las formas de la propiedad intelectual en su impronta caníbal y darnos espacio para nuevas construcciones, y para revalorar procesos colectivos que siempre han existido en las interacciones sociales donde se anclan los procesos de la mente.

\section{BIBLIOGRAFÍA}

Araya, Patricia, Meilipil, Juan, Núñez, Cecilia, Ossa, Claudio y Zárate Jorge, 2012: Estudio Exploratorio con Perspectiva de Género en Relación a los Derechos de Autor de los Creadores y Creadoras en Chile, Santiago, Departamento de Derechos Intelectuales de la Dirección de Bibliotecas, Archivos y Museos.

Arup, Christopher, 2009: "How are the different views of traditional knowledge linked by international law and global governance?", en Antons, Christoph (edit.), Traditional knowledge, traditional cultural expressions and intellectual property in the Asia-Pacific Region, Austin, Wolters Kluwer pp. 67-83.

BARTRA, Eli, 2005: Mujeres en el arte popular, México, UAM.

BARWA, Sharmishta y Rai, Shirin M., 2003: "Knowledge and/as power: A feminist critique of trade related intellectual property rights", Gender, Technology and Development, vol 7, $\mathrm{N}^{\circ} 1$ : pp. 91-113.

Boigeol, Anne, 2005: "Las Mujeres y la Corte. La difícil implementación de la igualdad de sexos en el acceso a la magistratura”, Academia. Revista sobre enseñanza del derecho, vol 3, $\mathrm{N}^{\circ}$ 6: pp. 3-25.

Bourdieu, Pierre, 2003: "Los juristas, guardianes de la hipocresía colectiva", Jueces para la democracia, $\mathrm{N}^{\circ}$ 47: pp. 3-5.

Brauneis, Robert y Oliar, Dotan, 2018: “An empirical study of the race, ethnicity, gender and age of copyright registrants”, The George Washington Law Review, vol 86, N²: pp. 101-156.

Burk, Dan L., 2007: "Feminism and Dualism in Intellectual Property", Journal of Gender, Social Policy and the Law, vol 15, No 2: pp. 183-206.

Burk, Dan L., 2015: "Diversity Levers”, Duke Journal of Gender Law E Policy, No 23: pp. 25-43.

Clance, Pauline Rose y Ament Imes, Suzanne, 1978: "The imposter phenomenon in high achieving women: Dynamics and therapeutic intervention", Psychotherapy: Theory, Research E Practice, vol 15, $\mathrm{N}^{\circ}$ 3: pp. 47-241.

Code, Lorraine, 1991: What Can She Know? Feminist Theory and the Construction of Knowledge, Ithaca, London, Cornell University Press.

Coоmbe, Rosemary J., 1998: The Cultural Life of Intellectual Properties. Authorship, Appropriation, and the Law, Durham and London, Duke University Press.

Craig, Carys J., Turcotte, Joseph F. y Coombe, Rosemary J., 2011: “What's feminist about open access? A relational approach to copyright in the academy", Feminist@law, vol $1 \mathrm{~N}^{\circ} 1$ : pp. $1-35$.

\footnotetext{
${ }^{71}$ Véase: IBARra, 2019. IBARra et al., 2020.
} 
Crenshaw, Kimberle, 1989: "Demarginalizing the Intersection of Race and Sex: A Black Feminist Critique of Antidiscrimination Doctrine, Feminist Theory and Antiracist Politics", University of Chicago Legal Forum, $\mathrm{N}^{\circ}$ 1: pp. 67-139.

Crenshaw, Kimberle, 1991: "Mapping the Margins: Intersectionality, Identity Politics, and Violence against Women of Color", Stanford Law Review, vol 43, N 6: pp. 99-1241.

Dommann, Monika, 2008: "Lost in tradition? Reconsidering the history of folklore and its legal protection since 1800", en Graber, Christph B. y Burri-Nenova, Mira (edit.), Intellectual Property And Traditional Cultural Expressions In A Digital Environment, Cheltenham, Edward Elgar, pp. 3-16.

Drahos, Peter, 1996: "Justifying intellectual property: back to the beginning”, en A Philosophy of Intellectual Property, Aldershot, Ashgate, pp. 13-39.

Drahos, Peter y Braithwaite, John, 2002: Information Feudalism: Who Owns the Knowledge Economy (London, Earthscan).

Edelman, Lauren B. y STRYKer, Robin, 2005: "A sociological approach to law and the economy", en Smelser, N.J y Swedberg, R. (edit.), The Handbook of Economic Sociology, New York, Rusell Sage Foundation, pp. 51-527.

Escobedo, Ezequiel, 2019: "Propiedad Intelectual de Pueblos Indígenas: Protección de los Textiles Tradicionales". Tesis para obtener el grado de Licenciado en Derecho por el Centro de Investigación y Docencia Económicas (CIDE).

Fernández De Calderón, Cándida, 2001: Grandes Maestros del Arte Popular Mexicano, México, Fomento Cultural Banamex.

Flórez, Margarita, 2007: "Can we protect traditional knowledge?", en Santos, Boaventura de Sousa (edit.), Another knowledge is possible. Beyond the northern epistemologies, New York, Verso, pp. 71-249.

Foucault, Michel, 1998: "What is an author?", en Preziosi, D. (edit.), The Art of Art History: A Critical Anthology, New York, Oxford University Press, pp. 299-314.

Freeman, Hadley, 2006: "Behind every great male writer...", The Guardian, disponible en: https:/www.theguardian.com/world/2006/mar/15/gender.books. Fecha de consulta: 19 de mayo de 2021.

Frietsch, Rainer, Haller, Inna, Funken-Vrohlings, Melanie y Grupp, Hariolf, 2009: "Gender-specific patterns in patenting and publishing", Research Policy, No 38: pp. 99-590.

GARrido, Eva María, 2015: "La artesanía como forma de expresión de tradiciones estéticas indígenas. El caso purhépecha”, en Pérez, S. (edit.), Artesanías y saberes tradicionales, Zámora, El Colegio de Michoacán, pp. 60-139.

Gearhart-Sema, Terra L., 2009: “Women’s work, women's knowing: intellectual property and the recognition of women's traditional knowledge", Yale Journal of Law and Feminism, vol 21, No 2: pp. 372-404.

Guarino, Cassandra M. y Borden, Victor M. H., 2017: "Faculty Service Loads and Gender: Are Women Taking Care of the Academic Family?", Research in Higher Education, vol 58, $\mathrm{N}^{\circ}$ 6: pp. 94-672.

Guerrilla Girls, 1989: "Do women have to be naked to get into the Met Museum?”, Guerrilla Girls’ Most Wanted: 1985-2006 (series).

Halbert, Debora, 2006: "Feminist interpretations of intellectual property", Journal of Gender, Social Policy and the Law, vol 14, No 3: pp. 60-431.

Hale, Charles R., 2006: "Activist Research v. Cultural Critique: Indigenous Land Rights and the Contradictions of Politically Engaged Anthropology”, Cultural Anthropology, vol 21, $\mathrm{N}^{\circ}$ 1: pp. 96-120.

Haunss, Sebastian, 2015: Conflicts in the Knowledge Society. The Contentious Politics of Intellectual Property, Cambridge, Cambridge University Press. 
Hoisl, Karin y Myriam Mariani, 2017: "It's a Man's Job: Income and the Gender Gap in Industrial Research", Management Science, vol 63, No 3: pp. 90-766.

Hunt, Jennifer, Jean-philippe Garant, Hannah Herman, y David J. Munroe, 2013: "Why are women underrepresented amongst patentees?", Research Policy vol 42: pp. 43-831.

IBARRA, Francisco Javier, 2012: "La formación jurídica en el siglo XXI", en Ibarra, Francisco Javier, Rojas, Ma. Ovidia, y Pineda, María Elena (edit.), La educación jurídica. Retos para el siglo XXI, México, UMSNH, Red de Sociología Jurídica en América Latina y el Caribe, pp. 75-101.

Ibarra, Lucero, 2016: "Políticas Culturales y Derechos Culturales a través de los Diablos de Ocumicho", Direito, Estado e Sociedade, No 48: pp. 196-223.

IbARra, Lucero, 2019: "Negociando la agenda epistémica del Derecho: la propiedad intelectual y políticas colectivas", en Tapia, S. M., Gómez, D.L. y Solano, V. (edit.), Estudios Jurídicos Críticos en América Latina, Cali: Dike, Universidad Santiago de Cali, pp. 87-106.

Ibarra, Lucero, Escobedo, Ezequiel y Fogata Kejtsitani, 2020: “What's yours is mine and what's mine is mine? Re-thinking intellectual property and research ethics from the experience of the Purhépecha community of Cherán”, Oñati Socio-Legal Series, vol 1, No 10: pp. 6-34.

Jaszi, Peter y Woodmansee, Martha, 1994: "Introduction", en Woodmansee, Martha y Jaszi, Peter (edits.), The Construction of Authorship. Textual Appropriation in Law and Literature, London, Duke University Press, pp. 1-13.

Jensen, Kyle, Kovács, Balázs y Sorenson, Olav, 2018: "Gender differences in obtaining and maintaining patent rights”, Nature Biotechnology, vol 36, No 4: pp. 9-307.

Kahler, Annette I., 2011: "Examining Exclusion in Woman-Inventor Patenting: A Comparison of Educational Trends and Patent Data in the Era of Computer Engineer Barbie", Journal of Gender, Social Policy and the Law, vol 19, N 3: pp. 98-773.

Lawson, Ashley, 2015: "The Muse and the Maker: Gender, Collaboration, and Appropriation in the Life and Work of F. Scott and Zelda Fitzgerald", The F. Scott Fitzgerald Review, vol 13, $\mathrm{N}^{\mathrm{o}} 1$, pp. 76-109.

Lista, Carlos Alberto, 2012: “¿Derecho sin justicia? Los déficit de la educación jurídica en la socialización de los abogados en Argentina” en Ibarra, Francisco Javier, Rojas, Ma. Ovidia y Pineda, María Elena (edit.), La educación jurídica. Retos para el siglo XXI, México, UMSNH, Red de Sociología Jurídica en América Latina y el Caribe, pp. 35-73.

MaCleOD, Morna, 2018: "Género, análisis situado y epistemologías indígenas: descentrar los términos del debate", en Leyva, Xochitls y Pascal, Cecile (edit.), Prácticas Otras de Conocimiento (s). Entre Crisis, entre Guerras. Vol. II, San Cristóbal, Cooperativa Editorial Retos, pp. 32-58.

Maliniak, Daniel, Powers, Ryan y Walter, Barbara F., 2018: "The Gender Citation Gap in International Relations", International Organization, vol 67, No 4: pp. 889-922.

Mallapaty, Smriti, 2018: "Paper authorship goes hyper", Nature Index. Disponible en: https:// www.natureindex.com/news-blog/paper-authorship-goes-hyper. Fecha de consulta: enero del 2019.

Manzo, Mariana A., Ibarra, Elena Alejandra, e Ibarra, Lucero, 2016: "Las mujeres en la justicia: discusiones desde el campo de los estudios socio-jurídicos”, en Manzo, Mariana A. y Rojas, M. Ovidia (edit), Profesión Jurídica: Discusiones sobre la Práctica del Derecho en América Latina y el Caribe, Morelia, UMSNH, Red de Sociología Jurídica en América Latina y el Caribe, pp. 25-57.

Masson, Sabine, 2018: "Transformar la investigación desde las prácticas feministas poscoloniales. De vuelta a mi experiencia etnográfica y activista con Tzome Ixuk”, en Leyva, Xochitl y Pascal, Cecile (edit.), Prácticas Otras de Conocimiento (s). Entre Crisis, entre Guerras. Vol. II, San Cristóbal, Cooperativa Editorial Retos, pp. 59-82. 
Mitchell, Kristina M. W. y Martin, Jonathan, 2018: "Gender Bias in Student Evaluations". PS: Political Science E Politics, $\mathrm{N}^{\circ} 215$ : pp. 1-5.

Mithlo, Nancy Marie, 2019: “'A Real Feminine Journey’: Locating Indigenous Feminisms in the Arts”, Meridians, vol 9, $\mathrm{N}^{\circ}$ 2: pp. 1-30.

Monroe, Kristen, Ozyurt, Saba, Wrigley, Ted y Alexander, Amy, 2008. "Gender equality in academia: Bad news from the trenches, and some possible solutions", Perspectives on politics, vol 6, No 2: pp. 215-233.

Montes, Fanny Ekaterina, 2020: "Katoü (Wayuu bag): a material axis of the relationship between Arijunas (non-Wayuu) and Wayuu people", Oñati Socio-Legal Series, vol 10, No 1, pp. 35-57.

Oehlerich De Zurita, Annie, 1999: Ni robo, ni limosna. Pueblos indígenas y propiedad intelectual, Santa Cruz, IBIS, CEJIS, CABI, CIDOB.

Paliyenko, Adrianna M., 2016: “Un/sexing genius”, Women Shaping French Poetic History, 1801 1900, USA, Penn State University, pp. 10-39.

Pateman, Carole, 1995: El contrato sexual, México, Anthropos, Universidad Autónoma Metropolitana - Iztapalapa.

Pozas, Andrea y Ríos, Julio, 2021: Enderezar el derecho. Por la regulacion de la educación y profesión jurídicas y contra la discriminación por género, México, CIDE, World Justice Project, Ilustre y Nacional Colegio de Abogados de México, Barra Mexicana, UNAM, ITAM ANADE, Mexicanos Contra la Corrupción y la Impunidad.

RAPPAPORT, Joanne, 2018: "Más allá de la observación participante: la etnografía colaborativa como innovación teórica”, en Leyva, Xochitl y Pascal, Cecile (edit.), Prácticas Otras de Conocimiento (s). Entre Crisis, entre Guerras. Vol. I, San Cristobal, Cooperativa Editorial Retos, pp. 52-323.

Ricoy, Rosa, 2015: “Teorías Jurídicas Feministas”, en Fabra, Á. Jorhe, Vaquero, Luis y Núñez (edit.), Enciclopedia de Filosofía y Teoría del Derecho. Vol. 1, México, UNAM, pp. 99-459.

Rosenblatt, Betsy y Rebecca Tushnet, 2015: "Transformative Works: young women's voices on fandom and fair use”, en BAIley, J. y Steeves, V. (edit.), eGirls, eCitizens. Putting Technology, Theory and Policy into Dialogue with Girls' and Ypung Women's Voices, Ottawa, University of Ottawa, pp. 385-409.

SANTA María Tlahuitoltepec - Autoridad Comunitaria, 2015: Tlabuitoltepec: Contra la apropiación de un patrimonio cultural, Oaxaca, Autoridad Comunitaria de Santa María Tlahuitoltepec.

Santamaría, Esteban y Castro de Ángel, Carlos Arturo, 2016: Panorama de la Propiedad Intelectual en México: Otra Perspectiva, México.

Sontag, Susan, 2007: At the same time, Nerw York, Farrar, Straus and Giroux.

SPENCE, Suzy y Brack, Leslie, 2009: "Womanhouse". Disponible en: http://www.womanhouse. net/website-mission. Fecha de consulta: 13 de agosto de 2019

SWANSON, Kara W., 2015: "Intellectual property and gender: reflexions on accomplisments and methodology", Journal of Gender, Social Policy and the Law, vol 24, No 1: pp. 98-175.

Teubner, Gunter y Fischer-Lescano, Andreas, 2008: "Cannibalizing epistemes: will modern law protect traditional cultural expressions”, en Graber, Chritpoh B. y Burri-Nenova, Mira (edit.), Intellectual Property And Traditional Cultural Expressions In A Digital Environment, Cheltenham, Edward Elgar, pp. 17-45.

Topaz, Chad M., Klingenberg, Bernhard, Turek, Daniel, Heggeseth, Briana, Harris, Pamela E., Blackwood, Julie C., Ondine, C., Nelson, Steven y Murphy, Kevin M., 2019: "Diversity of artists in major U.S. museums", PLoS ONE, vol 14, No 3: pp. 1-15.

Toro, David, 2019: "Los tejidos: los libros que la colonia no pudo quemar", Km. 169 Prensa Comunitaria. Disponible en: https://www.prensacomunitaria.org/los-tejidos-los-libros-quela-colonia-no-pudo-quemar/. Fecha de consulta: 25 de marzo de 2019. 
Treviño, Gloria María y coautoras, 2018: "Sentipensar con Tejedoras, Bordadoras, Brocadoras y sus Aliadas en los Altos de Chiapas cómo Proteger lo que les Pertenece”, Tesis para obtener el grado de Maestra en Antropología por el Centro de Investigaciones y Estudios en Antropología Social (CIESAS).

Vaidhyanathan, Siva, 2017: Intellectual Property. A Very Short Introduction, New York: Oxford University Press.

White, Kate, 2015. "Glass ceilings and monastic men: keeping women in science" Australian Quarterly, vol 86, N 3: pp. 14-25.

WIPO, 2017: Protect and Promote Your Culture. A Practical Guide to Intellectual Property for Indigenous Peoples and Local Communities, Geneva, WIPO.

Woodmansee, Martha, 1984: "The genious and the copyright: Economic and legal conditions of the emergence of the 'author'”, Eighteenth-Century Studies, vol. 17, No 4: pp. 48-405.

Woodmansee, Martha, 1994: "On the author effect: recovering collectivity", en Woodmansee, Martha y Jaszi, Peter (edit.), The Construction of Authorship. Textual Appropriation in Law and Literature, London, Duke University Press, pp. 15-28.

Zapata-Martelo, Emma y SuÁreZ-SAn RomÁn, Blanca, 2007: "Las artesanas, sus quehaceres en la organización y en el trabajo", Ra Ximbai, vol 3, N 3: pp. 591-620. 
